\title{
Intelligent loT-based large-scale inverse planning system considering postmodulation factors
}

\author{
Yihua $\mathrm{Lan}^{1} \cdot$ Fang $\mathrm{Li}^{2} \cdot{\text { Zijun } \mathrm{Li}^{3} \cdot \text { Binglei Yue }}^{4} \cdot{\text { Yin } \mathrm{Zhang}^{5}}^{(1)}$
}

Received: 30 July 2020 / Accepted: 22 September 2020

(c) The Author(s) 2020

\begin{abstract}
The model and algorithm of intensity-modulated radiotherapy (IMRT) are updated increasingly quickly, but the hardware upgrade of primary hospitals often lags behind. The new generation of intelligent precise radiotherapy platforms provides users with intelligent medical consortium services using big data, artificial intelligence and industrial Internet of Things technology. This technology can ensure that under the real-time guidance of a professional medical consortium, primary hospitals can realize rapid large-scale reverse planning design and can more accurately consider many factors of postprocessing. Although large-scale healthcare systems, such as volumetric-modulated arc therapy and other accurate radiotherapy technologies, have developed rapidly, the development of step-and-shoot-mode IMRT technology is still very important for developing countries. For software, in addition to the conformity of the dose distribution, the modulation speed, convenience and stability of the later dose delivery should also be considered in inverse planning. Therefore, this paper analyzes the main problems in conventional IMRT inverse planning, including the smoothing of the fluence map, the selection of the gantry angle and the dose leakage of tongue-groove effects. To address these issues, a novel Intelligent IoT-based large-scale inverse planning strategy with the key factors of the postmodulation is developed, and a detailed flow chart is also provided. The scheme consists of two steps. The first step is to obtain a relatively optimal combination of gantry angles by considering the dose distribution requirements and constraints and the modulation requirements and constraints. The second step is to optimize the intensity map, to smooth the map based on prior knowledge according to the determined angles, and to obtain the final modulation scheme according to the relevant objectives and constraints of the map decomposition (leaf sequencing). In an experiment, we calculate and validate the clinical head and neck case. Because of the special gantry angle selection, the angle combination is optimized from the initial equivalent distribution to adapt to the target area and protect the nontarget area. The value of the objective function varies greatly after the optimization, especially in the target area, and the target value decreases by approximately $10 \%$. On this basis, we smooth the fluence map by a partial differential equation with prior knowledge and a minimization of the total number of monitor units. It is also shown from the objective function value that the target value is essentially unchanged for the target area, while for the nontarget area, the value decreases by $16 \%$, which is very impressive.
\end{abstract}

Keywords IoT-based planning · Fluence map · Gantry angle · Tongue-groove effects · Tongue-groove effects · Partial differential equation $\cdot$ Total monitor units

Yin Zhang

yin.zhang.cn@ieee.org

1 School of Computer and Information Technology, Nanyang Normal University, Nanyang 473061, China

2 School of Mechanical and Electrical Engineering, Nanyang Normal University, Nanyang 473061, China

3 Cancer center of Jiujiang First people's Hospital, Jiujiang 332000, China

4 School of Information and Security Engineering, Zhongnan University of Economics and Law, Wuhan 430073, China

5 School of Information and Communication Engineering, University of Electronic Science and Technology of China, Chengdu, China 


\section{Introduction}

\section{Research object}

In the development process from IMRT to VMAT, accurate radiotherapy technology has been improved. Although the technical level has been greatly developed, the treatment benefit is very limited from the perspective of patients and doctors. In this context, the industrial Internet of Things has brought a ray of sunshine to the development of precision radiotherapy [1]. With the support of Internet of Things and cloud computing, the conventional large-scale IMRT health system takes a second leap forward in development, and the radiotherapy healthcare system can be realized in more primary hospitals with high quality [2].

\section{Research background}

Halcyon $^{\mathrm{TM}}$, a smart radiotherapy platform of Varian, was officially launched in China in 2019 [3]. The Halcyon system is the first medical linear accelerator to realize $100 \%$ image guidance in the world [4]. It offers the advantages of high integration, high automation and high intelligence [5]. It further promotes radiotherapy from conventional "precise radiotherapy" to "intelligent precise radiotherapy" [6]. As a new generation of smart radiotherapy platform [7], the Halcyon system can connect with the Varian smart cloud 4.0 radiotherapy platform and provide users with comprehensive intelligent connection services by using big data, artificial intelligence and industrial Internet of Things technology [8]. With the support of the large-scale healthcare platform, the Halcyon system can be deployed in the medical union, which ensures that the primary hospital can carry out treatment safely, normally and effectively under the realtime guidance of the specialist medical union, thus opening the era of the medical alliance radiotherapy cloud [9].

\section{Current problems}

With the continuous innovation and development of radiotherapy technology and equipment, the methods of cancer radiotherapy are becoming increasingly diverse [10], including three-dimensional conformal radiation therapy (3D-CRT), static intensity modulation radiation therapy (SIMRT), volumetric-modulated arc therapy (VMAT), helical tomotherapy (TOMO)-IMRT, and stereotactic body radiotherapy (SBRT) [11]. New technology and new equipment continuously improve the treatment of cancer patients. Advances in radiotherapy equipment, such as dynamic multileaf collimator with arc therapy, have resulted in more accurate irradiation. With the improvement of the function of the treatment planning system, more precise and optimized treatment plans can be formulated.
To meet the practical needs of clinical applications, the trend in current developments of the software and hardware systems of radiotherapy is to expand from a simple planning design to the entire process of radiotherapy [12]. Radiotherapy system software not only involves a treatment planning system but also unified planning and design for the information management of radiotherapy, the design of radiotherapy plans, the technical services of radiotherapy and the implementation of radiotherapy [13]. Thus, the efficiency, safety and reliability of radiotherapy and the convenience of technical support and services can be improved [14].

\section{The contributions of this article}

The complete inverse planning process involves complex system engineering, which mainly includes three modules: a data preparation module, a plan optimization module and a simulation evaluation module $[15,16]$. First, we need to acquire computed tomography (CT) medical images. After obtaining these image data, clinicians must delineate each CT image manually to assist the later 3D computer positioning process. At the same time, the physical dosimeter obtains the data of the effect of the cumulative dose of different parts irradiated by different intensities of radiation through photographic film and obtains the dose effect matrix by means of a simulation. Second, inverse planning requires a dose target model and fluence map decomposition model and a solution process. Inverse planning needs to consider the clinical variables, target dose requirements (achieve the prescribed dose) and nontarget dose constraints (inhibition the irradiation dose), as well as the number of beams, the direction of the beam, the intensity of the beam, the hardware requirements of the multileaf collimator and the requirements of doctors for the time of radiation and the number of monitor units of the irradiation machine. Third, doctors need to visualize the simulation results of the calculated radiotherapy plan and objectively evaluate the advantages and disadvantages of the plan according to the dose volume histogram.

A novel Intelligent IoT-based large-scale inverse planning strategy with the key factors of the postmodulation is developed, and the whole process is described with a pseudo code flow. The scheme consists of two steps. The first step is to obtain a relatively optimal combination of gantry angles by considering the dose distribution requirements and constraints and the modulation requirements and constraints. The second step is to optimize the intensity map and smooth the map based on prior knowledge according to the determined angles and then obtain the final modulation scheme according to the relevant objectives and constraints of the map decomposition. 


\section{Related work}

Volume-modulated radiotherapy (VMRT) has been widely used in the past 10 years, and to some extent, VMRT has proven superior to conventional intensity-modulated radiotherapy, such as modulation speed and dose conformal degree. However, VMRT has necessitated higher requirements for high-precision control, quality control and performance verification of radiotherapy equipment [17]. Such requirements make it too expensive for developing countries to upgrade the multileaf collimator. On the other hand, VMRT has not fundamentally changed the treatment principle of intensity-modulated radiation therapy. Further improvement in the optimal treatment scheme of IMRT can lead to a dose distribution of IMRT which approximates that of the volume modulation scheme. At the same time, conventional IMRT can be achieved only by relying on the conventional multileaf collimator and the conventional dose verification method [18]. The economic benefits cannot be underestimated.

Conventional intensity-modulated radiation therapy involves the step-and-shoot mode $[19,20]$. To avoid the back and forth movement of the leaves leading to leaf wearing, sliding windows are usually used in clinical applications. Because static IMRT chooses a limited field to achieve dose modulation, the total numbers of monitor units in the modulation process of the intensity maps obtained by choosing different treatment fields are quite different. Because of the convex and concave physical characteristics of the blade, the tongue and groove effect in the modulation process is also a very important factor [21].

For radiotherapy of a particular type or organ, the doctor usually uses empirical field direction selection [22]. Although the field direction chosen in this way is more representative, the empirical field direction may not be the best choice because of the change of the target location. Therefore, the choice of the direction of the field is the first key problem in the radiotherapy planning system [23]. The conventional criterion for selecting the direction of the field is to ensure that the dose modulation distribution achieved by the fluence map can be optimized, ignoring the total number of monitor units and the tongue and groove effect in the modulation process [24].

In fact, the difference in the dose modulation distributions in a clinic with the objective of ensuring an optimal fluence map does not lead to an essential difference in clinical treatment $[25,26]$. In contrast, the total number of monitor units and the tongue and groove effect in the modulation process will lead to a great difference in the quality assurance and verification process of the entire radiotherapy process. In other words, the fluence maps that appear to have similar dose distribution states will produce distinct dose distribution states because of the variable difficulty in the subsequent dose mod- ulation process [27]. This is a major problem that must be considered in inverse planning [28]. The tongue and groove effect is the direct cause of the hot and cold points of a dose. To avoid this effect, it is necessary to avoid double or multiple peaks in the intensity maps: that is, to keep the high dose areas connected as much as possible. From a mathematical point of view, convex constraints cannot be used for the description. Through analysis, we can obtain a priori knowledge regarding the upper left blade and the right lower blade in the adjacent blades, or determine that the lower left blade and the right upper blade cannot collide, but should maintain a convex shape as much as possible during the sliding process $[29,30]$.

\section{Key parts of the radiotherapy software system}

The main hardware of radiotherapy includes an accelerator, a multileaf collimator, and a radiotherapy simulator. The radiotherapy software system mainly consists of a treatment planning system, database management system, treatment control system and radiotherapy simulation and verification system. Data exchange between systems is carried out through a high-speed network connection. The complete radiotherapy system acquires image data from external image acquisition equipment (such as $\mathrm{CT}$ and MRI) through a gigabit network. The composition and main functions of each system in the radiotherapy system are described below.

\section{Radiotherapy treatment planning system}

The treatment planning system obtains current patient image data from external image acquisition equipment, such as a CT machine, through a high-speed network. According to these image data and preset physical parameters of the therapeutic accelerator machine, the current treatment plan of patients is formulated, and the relevant data are sent to the database system. The main contents of the treatment plan include the parameters of the gantry, the parameters of the treatment bed, the multileaf collimator parameters, the treatment grading parameters and the radiation dose distribution parameters. A well-made treatment plan and patient-related image data will be sent to the database system in the form of digital imaging and communications in medicine (DICOM) files.

\section{Radiotherapy database management system}

The radiotherapy database management system consists of three parts: a database management program, a patient information database and a patient data file. The database management program is mainly used to create DICOM files and generate and manage database records of patient information. When the treatment planning system completes the

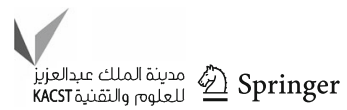


treatment plan, the content of the plan and the related image data will be sent to the database system in the form of DICOM files, which are stored in the patient information database by the database management program according to the DICOM protocol. When the IMRT system carries out the image acquisition process, an X-ray image and a CT image will also be sent to the database system through the network in the form of DICOM files. The patient information database is mainly used to store basic patient information, treatment plan data and patient-related image data. The basic information of the patients and the parameters of the gantry and the bed are obtained from the treatment plan file through the database management program. This information will be added to the relevant tables of the patient information database by the database management program. After the DICOM image data files and plan data files related to patients are stored in the patient data folder, their storage locations will also be recorded in the patient information database. Patient data files keep DICOM image data files and plan data files that are related to all patient treatment plans. The database management program extracts DICOM image data files and treatment plan data files and stores them separately. The specific storage locations will be stored in the related tables of the patient information database in the form of database records. X-ray images and CT image data will also be stored in the corresponding patient data file.

\section{Radiotherapy control system}

The control system is connected to the database system through the network. It can directly obtain the patient information and treatment plan data in the patient information database. The treatment control system downloads the treatment plan data related to the patient from the patient information database according to the currently selected information. When the treatment control system obtains the basic information and treatment plan data of the current patients from the patient information database according to the doctor's choice, the treatment control system will send start-up instructions to the IMRT system through the network. When the treatment control system receives the response message of the IMRT system, the treatment control system sends the basic information of the current treatment patients to the IMRT system. The IMRT system will use this information as an index to search the current treatment plan data, related image data and plan data file storage locations in the patient information database through the network and download them to the computer. When the IMRT system completes the position correction operation of the treatment bed through registration, the treatment control system will control the accelerator and multileaf collimator for the treatment of patients according to the current patient treatment plan data acquired previously. After all treatment operations are completed, the information of the treatment results is stored in the patient information database through the network.

\section{Radiotherapy simulation and verification system}

The radiotherapy simulation and verification system completes the DICOM interface with the radiotherapy planning system and radiotherapy control system and feeds the information of the reset and verification back to the database management subsystem. The simulation and verification system of radiotherapy can realize the functions of cone beam $\mathrm{CT}$ reconstruction and a $3 \mathrm{D}$ simulation and verification of the plan.

\section{Methodology of the large-scale planning system}

\section{Simplicity and controllability of the modulation by fluence map smoothing}

Smoothing technology is used to reduce the complexity of the fluence map, which is similar to image denoising in image processing. Fluctuations in the fluence map have a great influence on leaf sequencing, especially for the high-frequency region. Therefore, intuitively, if there are fewer fluctuations in the fluence map, the subsequent decomposition process based on a multileaf collimator will become much easier. The conventional smoothing methods include a smoothing strategy outside of the objective function, an implicit smoothing strategy in the objective function and an explicit smoothing strategy in the objective function.

The outside strategy adopts a low-pass filter to smooth the optimized fluence map directly. Linear filters are commonly used; typically, a linear convolution is used along the line direction. The main consideration is that the lead blades of the multileaf collimator are modulated by moving along the line direction. $x_{i, j, k}^{\prime}=\sum_{\lambda=-L}^{R} c_{\lambda} \cdot x_{i, j+\lambda, k}$, where $c_{\lambda}$ are the convolution coefficients and $x$ is the intensity, and $L$ and $R$ represent the lengths of the convolution domain areas. The main advantages of the smoothing strategy outside of the objective function are that the postprocessing is simple, the convolution coefficient is flexible, and the fluence map can be adjusted according to different filtering requirements. The disadvantage is that the smoothed results may seriously violate the prescription dose requirements.

The implicit strategy directly defines the specific smoothing penalty function, such as the curvature function (Eq. 1), L1-norm function (Eq. 2) or L2-norm (Eq. 3), where $x$ is the intensity, $m$ and $n$ are the intensity map size, and $l$ is the beam number. 


$$
\begin{aligned}
F(X)= & \frac{2 x_{i, j, k}-x_{i, j-1, k}-x_{i, j+1, k}}{\left(1+\frac{1}{4}\left(x_{i, j-1, k}-x_{i, j+1, k}\right)^{2}\right)^{2}} \\
F(X)= & \sum_{i=1}^{l} \sum_{j=2}^{m} \sum_{n=2}^{n}\left(\left|x_{i, j, k}-x_{i, j-1, k}\right|\right. \\
& \left.+\left|x_{i, j, k}-x_{i, j, k-1}\right|\right) \\
F(X)= & \sum_{i=1}^{l} \sum_{j=1}^{m-1} \sum_{n=1}^{n-1}\left(\left(x_{i, j, k}-x_{i, j+1, k}\right)^{2}\right. \\
& +\left(x_{i, j, k}-x_{i, j, k+1}\right)^{2} \\
& +\frac{1}{2}\left(x_{i, j, k}-x_{i, j+1, k+1}\right)^{2} \\
& \left.+\frac{1}{2}\left(x_{i, j, k}-x_{i, j+1, k-1}\right)^{2}\right)
\end{aligned}
$$

The advantage of the implicit strategy is that it reduces the smoothing postprocessing and secondary optimization calculation, and the model design is more concise. The disadvantage is that the design of the penalty function varies greatly. For a model that is easier to calculate, the designed penalty function is often relatively simple, which results in neglecting the other radiotherapy factors and a lack of flexibility in smoothing postprocessing.

The explicit strategy obtains the smoothed intensity map through low-pass filtering. On this basis, an optimization calculation with the objective function is carried out again to add a smoothing penalty function $\sum_{i=1}^{I} \sum_{j=1}^{m} \sum_{k=1}^{n} w_{i, j, k}$. $\left(x_{i, j, k}-x_{i, j, k}^{\prime}\right)^{2}$ to the objective item of the original fluence map optimization model. Quadratic optimization with feedback of the smoothing results of the intensity maps allows the reverse plan to meet the prescription dose distribution requirements to the greatest extent, and the calculation process is simple and clear. The disadvantage is that the term of the smoothing penalty function describes the difference between the actual fluence map and the smoothed fluence map. The intensity maps calculated by this strategy are often not optimal in terms of dose distribution.

Directional filtering based on a partial differential equation (PDE) is an effective image denoising method. On the other hand, denoising based on nonlocal means (NLM) provides the possibility of smoothing based on prior information. NLM makes use of redundant information in the image, which can retain the details of the image as much as possible while smoothing. The estimated value of the current pixel is obtained by a weighted averaging of the pixels in the image with similar neighborhood structure. The smoothing value of the pixel to be smoothed in the NLM is determined by a similarity measure between the local and global image blocks around the pixel. The algorithm makes full use of prior knowledge of the fact that image blocks in natural images are highly repeatable. The similarity is defined as $w(x, y)=$ $\exp \left(-\frac{\|V(x)-V(y)\|^{2}}{h^{2}}\right) / \sum_{y} \exp \left(-\frac{\|V(x)-V(y)\|^{2}}{h^{2}}\right)$. The original intensity smoothing algorithm is generally an isotropic smoothing strategy, and according to NLM denoising, we can introduce the prior knowledge mentioned above.

Therefore, the method of smoothing the fluence map can employ NLM to make full use of prior knowledge on the basis of PDE smoothing: that is, to limit the smoothing direction by considering not only the projection direction of the target area in the direction of the irradiation field but also the smoothing direction of the reducing MU.

\section{The influence of the monitor unit with different gantry angle selections}

To illustrate the problems arising from the selection of the beam angles, we provide the following test examples (Fig. 1). We assume that the prescription dose for the target area is 10 and that the dose of organ at risk (OAR) is no more than 5. A typical selection of the two perpendicular angles for the irradiation field is shown in the figure. For the combined field of angles 1 and 2, the total number of monitor units is 15 , and the dose of OAR is 5 . For the combined field of angles 3 and 4, the total number of monitor units is 20 , and the OAR dose is 0 . Thus, we can see that the optimization degree of the clinical dose distribution is inconsistent with the total number of monitor units when some target areas surround the distribution of OAR. For a certain optimization model, the value and direction of the angle of the irradiation field also need to be included in the complete optimization process.

\section{The influence of the dose modulation with different fluence maps}

IMRT is usually modulated by a multileaf collimator. For most target areas of different shapes and sizes, only a few leaves are in the range of the effective irradiation field, while the other blades outside the range of the effective field should be combined in pairs to prevent radiation leakage. However, to avoid the mechanical damage caused by the relative collision of the paired blades, a small clearance is usually included. A minimum external rectangular field must be specified for the conventional treatment collimator of the accelerator. This can not only shield the intermittent leakage radiation from each pair of the incompletely closed blades in the field but also shield the small external rectangular field between adjacent leaves. The corresponding control data are transmitted to the corresponding control system to realize the automatic setting of the minimum rectangular field and the multileaf collimator(MLC) effective field.

Due to the motion constraints of the upper and lower leaves of the multileaf collimator, it is generally required that the

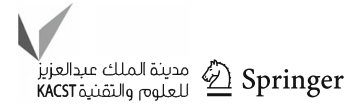


Fig. 1 The fluence maps of two typical irradiation fields with two vertical angles

\section{Fluence map of angle 2}
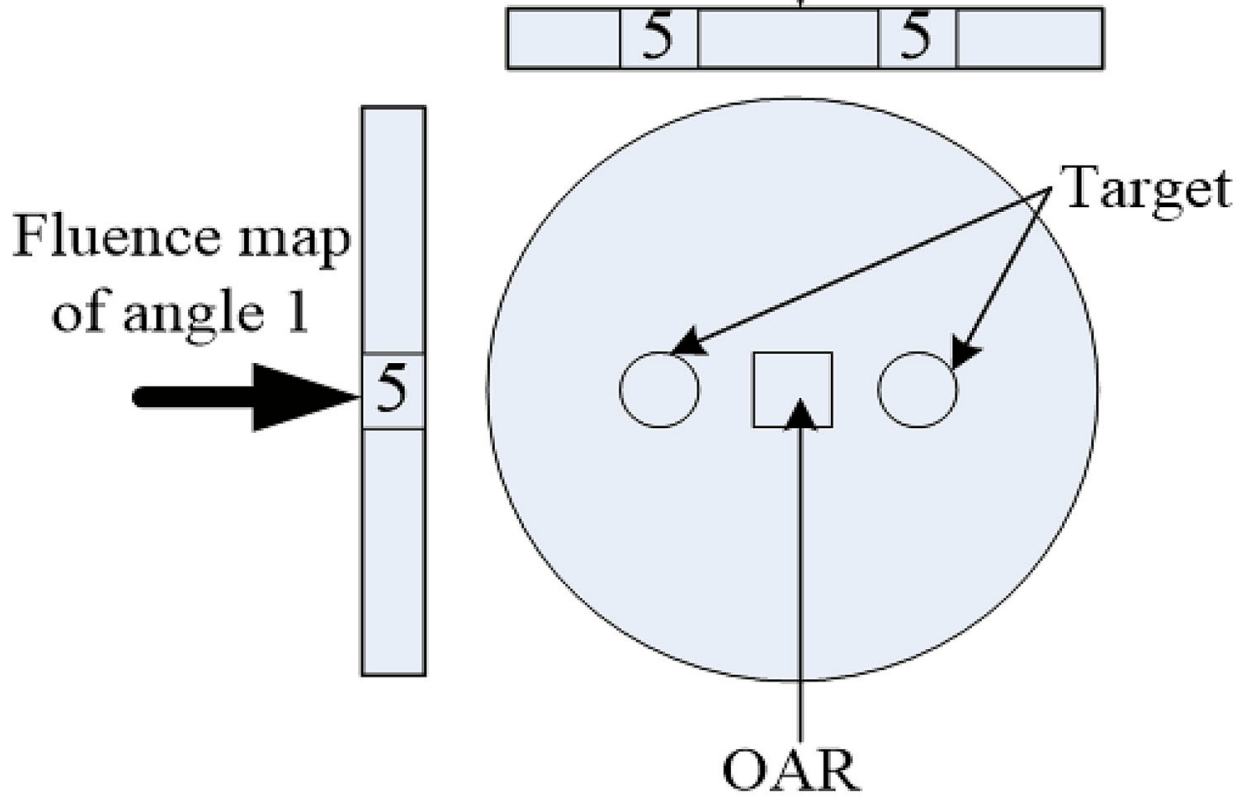

(a) The optimal fluence maps with a $0^{\circ}$ beam and a $90^{\circ}$ beam

\section{Fluence map of angle 3}

Fluence map

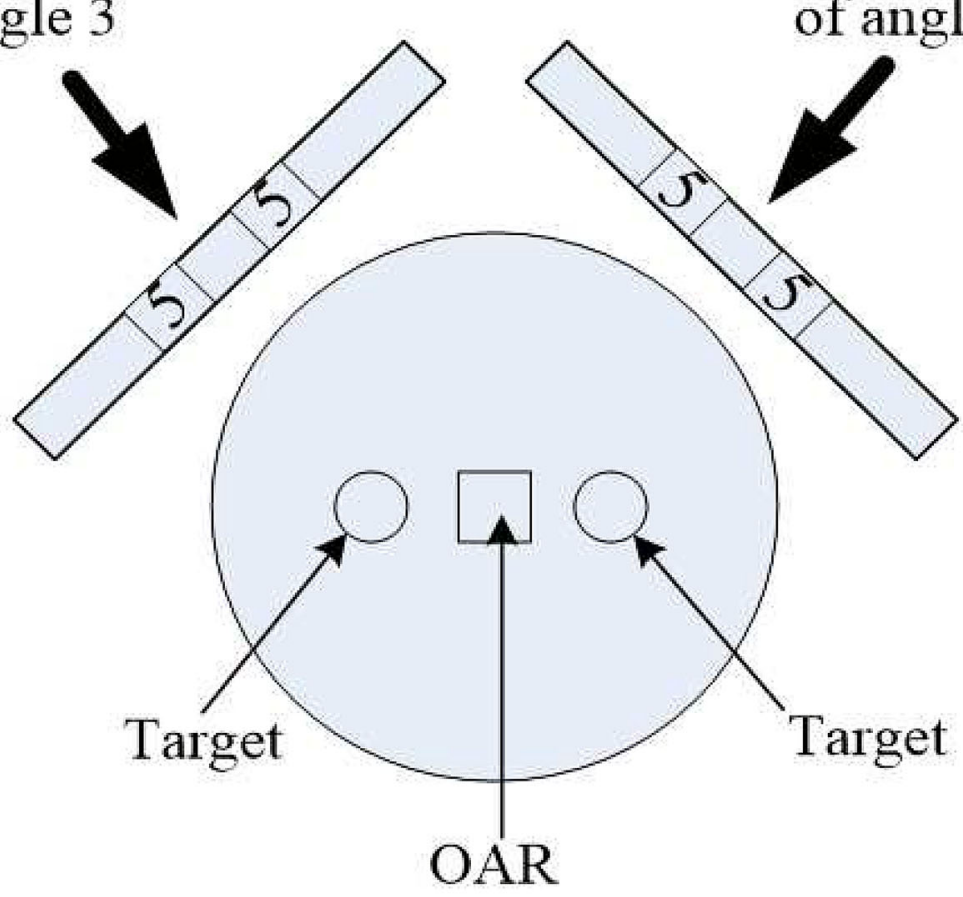

(b) The optimal fluence maps with a $-45^{\circ}$ beam and a $45^{\circ}$ beam 
Fig. 2 Typical two-step modulation strategy for the intensity map in a diagonally isolated distribution (the upper left figure is the first step of modulation, the upper right one is the second step, and the lower part is the modulation result)
Fig. 3 Collision by the single modulation when the fluence map is diagonally distributed (the left image shows the modulation process without collision constraint of adjacent leaves, and the right figure shows the modulation result)
Step1

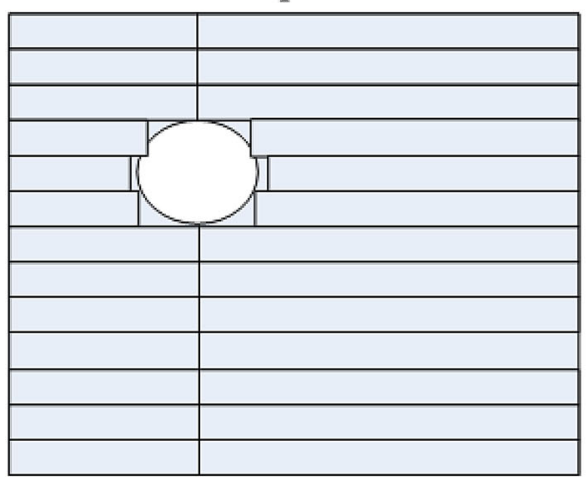

Step2
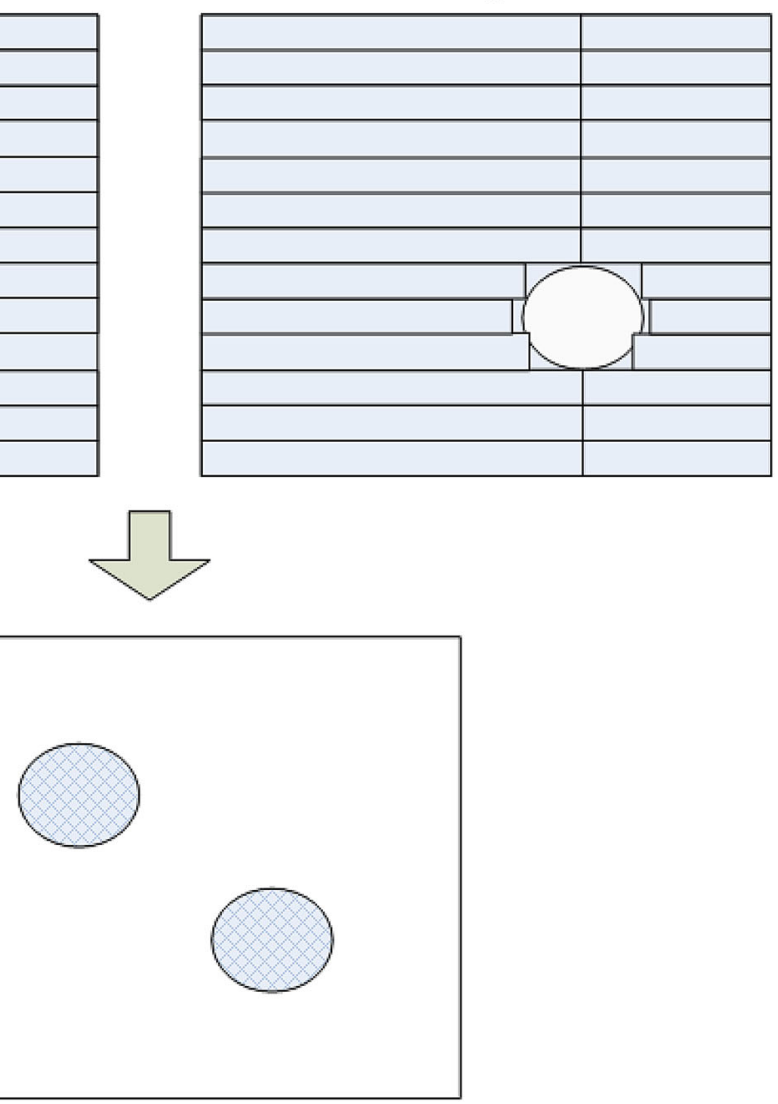

Step1
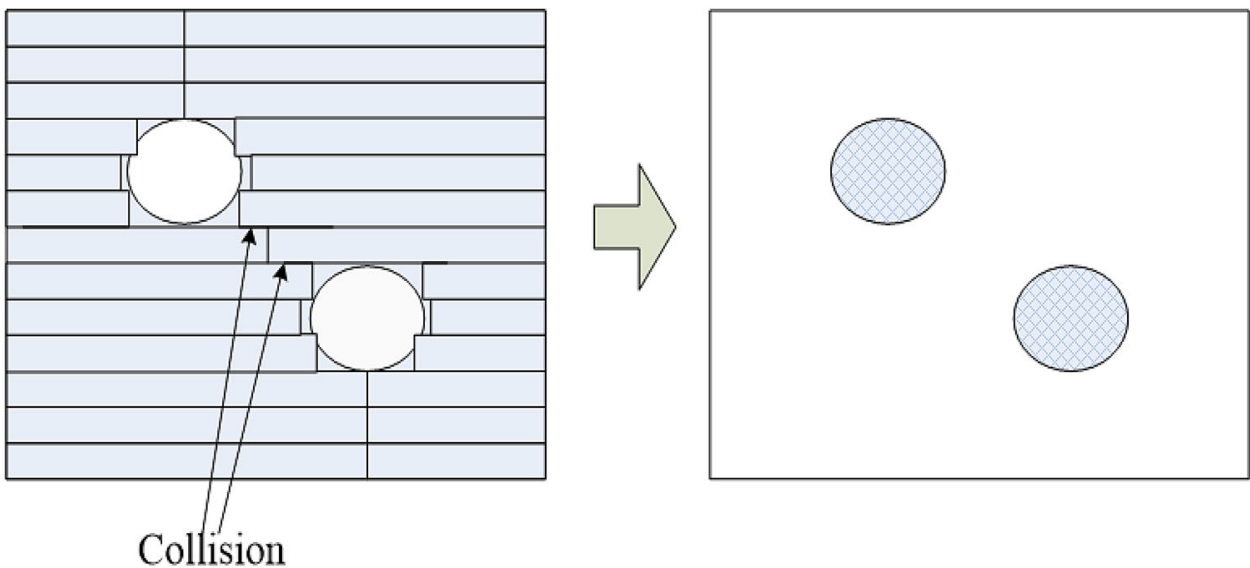

upper and lower leaves should not overlap with each other. Therefore, for the bimodal fluence map in the figure, a twostep modulation is usually needed (Fig. 2). If a single-step modulation is used, a collision between the upper and lower adjacent leaves will inevitably occur (Fig. 3). However, if the position of the bimodal intensity map is corrected, as shown in Fig. 4, it may be achieved by a one-step modulation. A onestep modulation means that the modulation process can be realized with less TNMU. It can be determined that to reduce the number of monitor units, and at the same time avoid a collision of the leaves, the optimization of the fluence map must also take this demand into account.

The design of the leaf section shape is an important factor that must be considered in a multileaf collimator. To ensure the minimum leakage dose between adjacent leaves and relative leaves when they are closed, the sides of the leaves should have convex grooves. The positions of the grooves can be machined in the middle of the leaf. However, this 
Fig. 4 There is no leaf collision when the fluence map is correlated up and down (the left image shows the modulation process with collision constraint of adjacent leaves, and the right figure shows the modulation result)

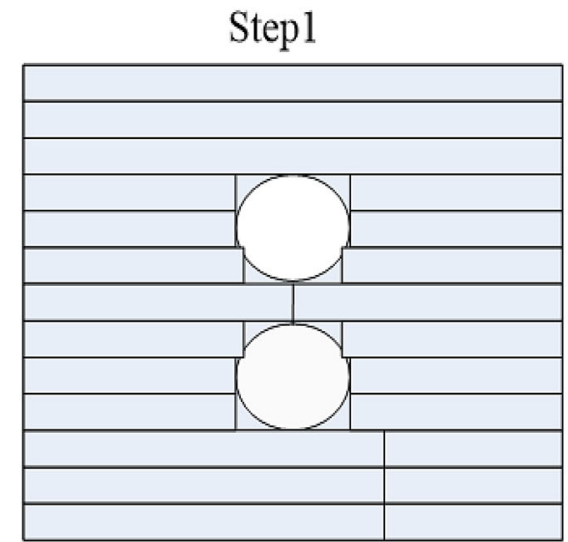

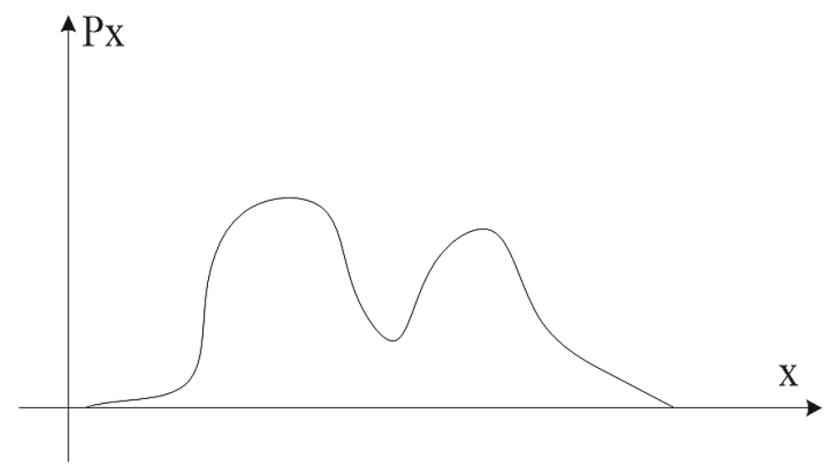

Fig. 5 A sketch of the fluence map projection

kind of structure requires a high processing accuracy. Sometimes, leaves fail because of the high resistance to motion. Many manufacturers have used step structures to produce the leaf. The tongue and groove effect is an unavoidable problem when the multileaf collimator is used for the dose modulation, which will lead to an underdose when the effect is serious.

\section{The solution strategy proposed in this paper}

\section{The complete process of inverse planning software}

In summary, in addition to the clinical dose requirement, many factors need to be considered in the complete dose modulation process because these factors exert a great influence on the simplicity of the dose modulation process, the stability of the dose distribution in the modulation process, and the possible dose leakage during the modulation process. Therefore, the smoothness of the intensity map, the selection of the field angle, the optimization of the dose intensity map, the reduction in the total machine hops, the number of subfields and the reduction in the tongue-groove effect should be taken into account as much as possible in the early stage.

The entire inverse optimization process is mainly divided into two steps. The first step is to obtain a relatively optimal combination of gantry angles by considering the dose distribution requirements and constraints and the modulation requirements and constraints. The second step is to optimize the intensity map and smooth the map based on prior knowledge according to the determined angles, and then to obtain the final modulation scheme according to the relevant objectives and constraints of the map decomposition (leaf sequencing). The whole process is described in programming language as follows.

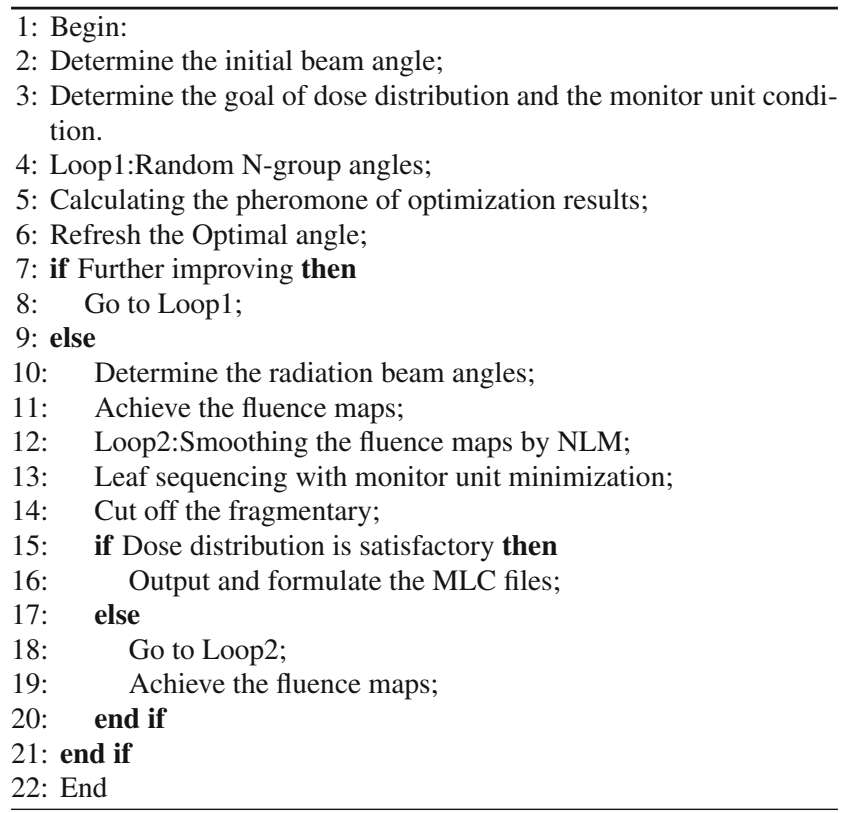

\section{Optimal angle combination by a finite beam angle}

First, the initial frame angle is given, and the dose distribution requirement and the postmodulation requirement are given by the big data through the Internet of Things. In this step, through an ant colony algorithm, the constraints and optimization objectives and iteration steps are set to determine 
Fig. 6 The outline of the target area and OAR for the test case

Fig. 7 Gantry angle comparison after the optimization
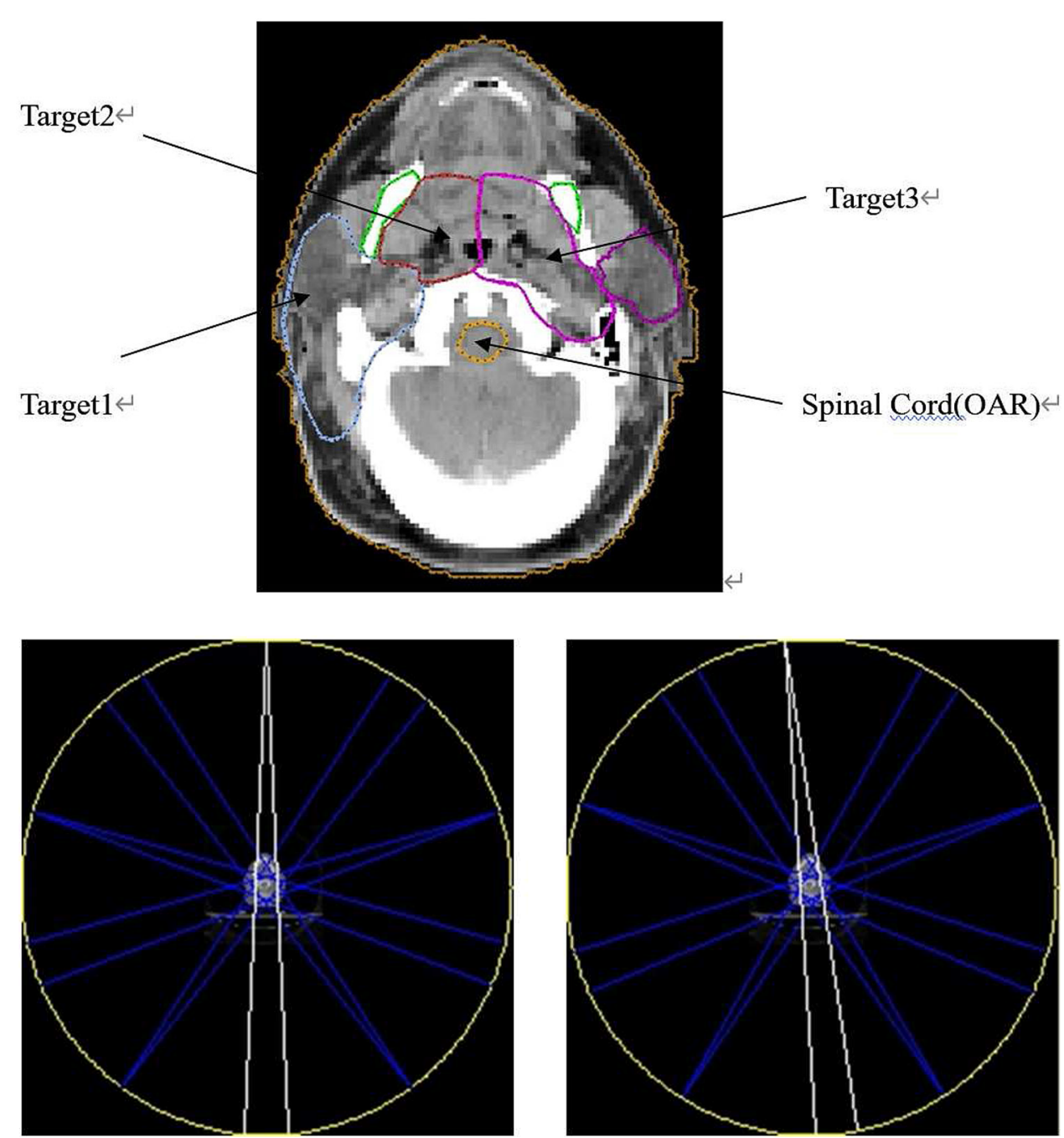

the optimal firing angle. We employ fast field selection based on a heuristic algorithm. The [31] ant colony algorithm is a typical heuristic intelligent algorithm. The ant colony algorithm is a bionic algorithm inspired by the foraging behavior of ants in nature. In nature, ant colonies can always find an optimal path from nests and food sources in the process of foraging. Thus, we consider that a group of individuals exhibit intelligent behavior through collaboration, which provides the possibility for solving complex problems. The ant colony algorithm is a probabilistic algorithm that is used to find the optimal path. We consider using this algorithm to filter the angle of the field. Here, we provide the definition of pheromones.

The fluence map is projected in the $X$ direction, and we can obtain the projection curve in the $X$ direction $p_{x}$ (Fig. 5).

Then, a parameter is defined to describe the overall distribution of the intensity maps. The quality of the intensity maps is not a measure of the complexity of the intensity maps, but mainly considers the graded complexity of the dose modulation process. Because of the directionality of
Table 1 Dose requirements for the head and neck case

\begin{tabular}{llll}
\hline Target area & 1 & Target 1 & $70 \mathrm{~Gy}$ \\
& 2 & Target 2 & $64 \mathrm{~Gy}$ \\
Nontarget area & 3 & Target 3 & $60 \mathrm{~Gy}$ \\
& 1 & Brain stem & Maximum 54 Gy \\
& 2 & Parotid gland & Maximum 70 Gy \\
& 3 & Mandible & Maximum 73 Gy \\
& 4 & Spinal cord & Maximum 45 Gy \\
& 5 & Other tissue & Maximum 75 Gy \\
\hline
\end{tabular}

the multileaf collimator leaf motion, the grading complexity needs to be considered according to the direction of the leaf motion. Thus, we propose a definition $\eta=\frac{\sum \Delta p_{x}^{+}}{\int p_{x} \mathrm{~d} x}$, where $p_{x}$ is the fluence map projection as shown in Fig. 5, $\Delta p_{x}^{+}$is the positive term after difference. This parameter represents the fluctuation characteristics in the $X$ direction. Obviously, the large-area average irradiation of a single peak will lead to a reduction in this parameter as much as possible. Then, the 
absolute value of the difference in the variance between the projection function in the $X Y$ and $Y X$ directions is calculated to prevent the tongue-groove effect, which is defined as $\theta=\left|\sigma_{x y}-\sigma_{y x}\right| \cdot\left(\sum \sum X\right)$. Considering the objective function value of the dose distribution and the total number of monitor units, the pheromone of any fluence map is defined as

$\phi=\alpha \cdot \eta+\beta \cdot \theta+\gamma \cdot$ objValue $+\lambda \cdot T N M U$,

where $\alpha+\beta+\lambda=1, \eta$ is the smoothness parameter, $\theta$ is the tongue-groove parameter, objValue is the dose conformity parameter, and $T N M U$ is the irradiation duration parameter.

\section{Smoothing fluence map by a PDE with prior knowledge}

Linear diffusion filters blur useful structural information while denoising; consequently, confusion of the structural information occurs. Therefore, we present the smoothing algorithm by a PDE with prior knowledge. Nonlinear heterogeneous diffusion equations require minimization of the gradient energy. We set the gradient energy function as $E(u)=\int|D u(x, y)| \mathrm{d} x \mathrm{~d} y$.

The objective is to solve the zero-flow boundary variational problem $\left\{\begin{array}{l}J(u)=\min E \\ u \in\left\{u \in C^{2}(\Omega)|u|_{\partial \Omega}=0\right\}\end{array}\right.$ by using Green's formula, where Euler's necessary conditions and the zero-flow boundary conditions are as follows.

$$
\int \Omega\left[\frac{\partial F}{\partial u^{*}}-\frac{\partial}{\partial x}\left(\frac{\partial F}{\partial u_{x}^{*}}\right)-\frac{\partial}{\partial y}\left(\frac{\partial F}{\partial u_{y}^{*}}\right) \mid v \mathrm{~d} x \mathrm{~d} y=0\right.
$$

The solution satisfies the heterogeneous diffusion equation $\partial u / \partial t=\operatorname{div} \frac{D u}{|D u|}$.

If we set the energy function as

$$
\begin{aligned}
E(u) & =\int g(v)|\nabla u(x, y)|+\beta\left(u-u_{0}\right)^{2} \mathrm{~d} x \mathrm{~d} y, v \\
& =D\left(G_{\sigma}^{*} u\right)
\end{aligned}
$$

the corresponding heterogeneous diffusion equation is as follows.

$$
\begin{aligned}
\partial u / \partial t= & g(v)|D u| \operatorname{div} \frac{D u}{|D u|}+D g(v) g D u \\
& -\beta|D u|\left(u-u_{0}\right)+\left(\frac{\partial g(v)}{\partial u_{x}}, \frac{\partial g(v)}{\partial u_{y}}\right) \\
& g|D u| \operatorname{div}|D u|+\operatorname{div}\left(\frac{\partial g(v)}{\partial u_{x}}, \frac{\partial g(v)}{\partial u_{y}}\right) g|D u|^{2} .
\end{aligned}
$$

We set the fluence map as the image to be denoised. Anisotropic diffusion occurs with the above function according to the vertical direction of the MLC leaf movement and other related directions, and the projection direction of the OAR projection on the projection field is also considered.

\section{Leaf sequencing while minimizing total number of monitor units}

For the $i$-th fluence map $\overline{\boldsymbol{X}}_{i}$, we assume that $\boldsymbol{I}_{i}^{T}$ and $\boldsymbol{I}_{i}^{L}$ are the leaf trajectory matrices of the trailing and precursor, respectively. When the last rear-drive leaf covers the last beam block in the intensity map, the entire modulation process of the intensity map of the field ends. Therefore, the monitor unit of this fluence map is $\mathrm{MU}_{i}=\max _{j}\left\{\boldsymbol{I}_{i}^{T}(j, n)\right\}$. The total number of monitor units is TNMU $=\sum_{i=1}^{l} M U_{i}=$ $\sum_{i=1}^{l}\left[\max _{j}\left\{\boldsymbol{I}_{i}^{T}(j, n)\right\}\right]$. If we add constraints to the dose model, which represent the upper bound of the total number of monitor units, then we form a map optimization model with the total machine hop constraints for the MLC modes.

Dose distribution objective function in target area and OAR area S.t.

$$
\left\{\begin{array}{l}
\text { Doseconstraintsintargetandnon }- \text { targetareas } \\
0 \leq x \\
x_{i, j, 0}=0, i=1,2, \ldots, l ; j=1,2, \ldots, m \\
\sum_{i=1}^{l}\left[\max _{j} \sum_{k=1}^{n}\left\{\max \left\{\left(x_{i, j, k}-x_{i, j, k-1}\right), 0\right\}\right\}\right] \leq T N M U_{0} .
\end{array}\right.
$$

\section{Design of the experiments}

The computer used has an Intel $2.13 \mathrm{GHz}$ Pentium P6200 CPU with 2 GB of memory. The software package Computational Environment for Radiotherapy Research (CERR), developed and published by the University of Washington, USA, is used as the inverse planning design platform of IMRT. The calculation of the intensity matrix in our experiment is based on the QIB dose calculation engine, which is also a part of IMRTP in the CERR development platform. All test cases are public cases downloaded from the website published by CERR. The prescription dose of the target area and dose constraints of the nontarget area are shown in the table as follows (Table 1).

\section{Experimental results and analysis}

As shown in the figure, the selection of the gantry angle should be adapted to the distribution of the target area and the OAR because the target area is semicircumferential to the OAR (Fig. 6). 
Table 2 The objective value and monitor unit comparison after the angle optimization

\begin{tabular}{lll}
\hline & Initialized gantry angles & Optimized gantry angles \\
\hline Angle value & {$[0,72,144,216,288]$} & {$[354,70,147,212,290]$} \\
Objective value & 112.2340 & 100.7484 \\
Target value & 37.6569 & 27.9765 \\
Nontarget value & 74.5770 & 72.7720 \\
Monitor units & {$[28,18,34,21,10]$} & {$[22,19,35,25,11]$} \\
\hline
\end{tabular}
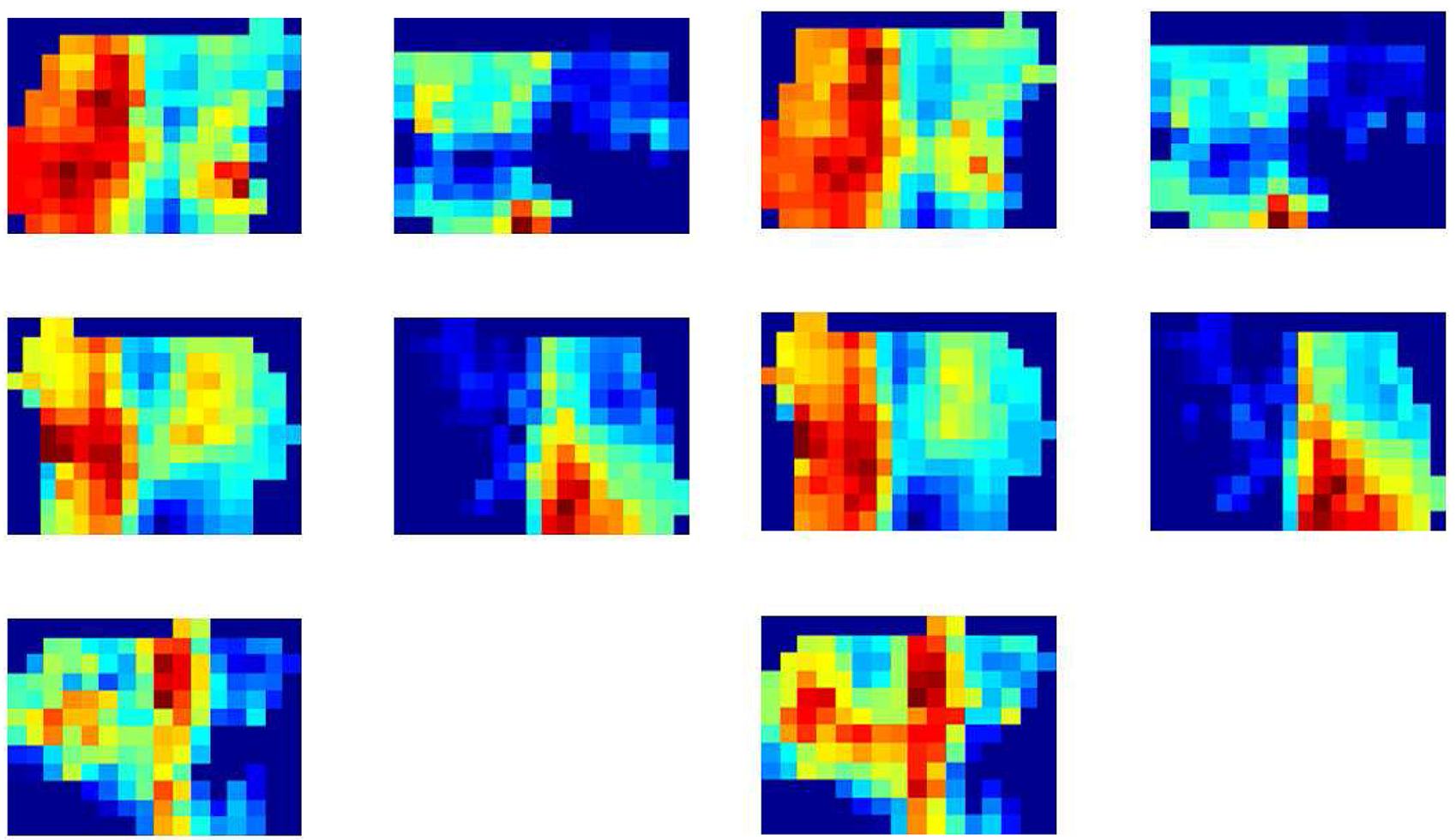

Fig. 8 Fluence map comparison between the initial (left) and optimized (right) gantry angles
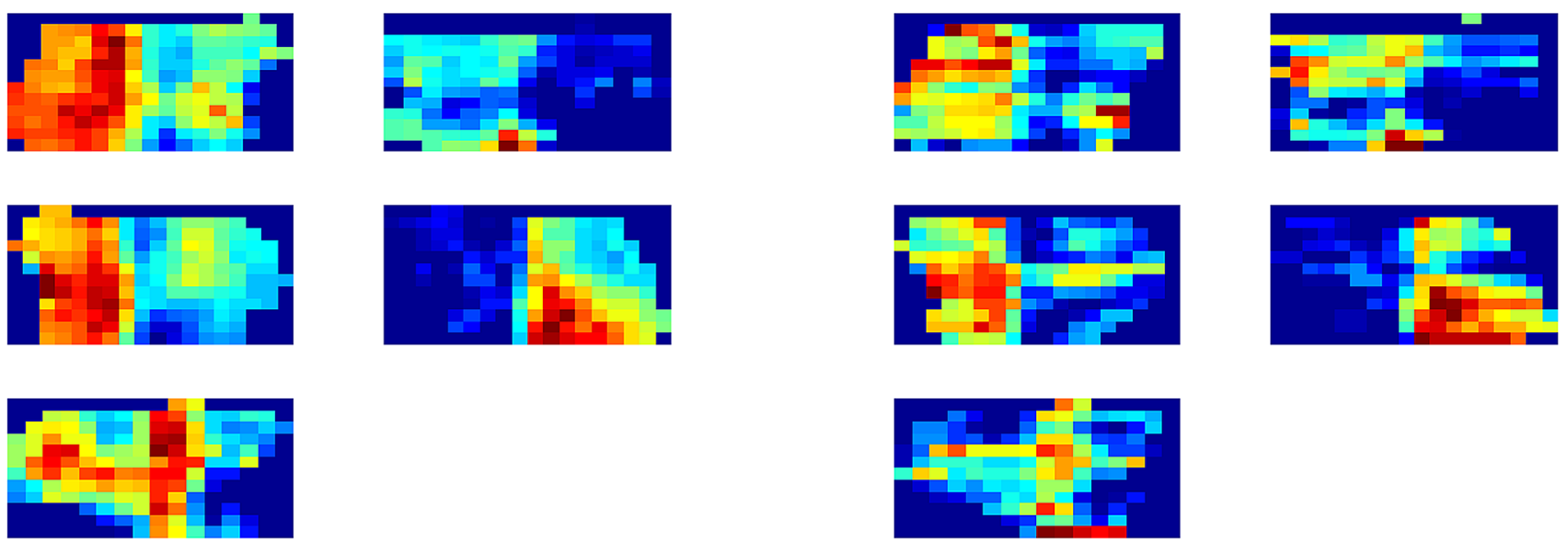

(a)

(b)

Fig. 9 Fluence map comparison between conventional smoothing (a) and PDE-based smoothing (b) 
In the experiments, the smoothness parameter is set as 0.2 , the tongue-groove parameter is set as 0.2 , the dose conformity parameter is set as 0.5 , and the irradiation duration parameter is set as 0.1 . That is, we are more concerned about the dose conformity. After the gantry angle selection (Fig. 7), we can obtain a better result.

As shown in Table 2, because a higher weight is set for the dose conformity, and the smoothness and tongue-groove effects are taken into account, the weight of the TNMU is lower; therefore, after the angle optimization, the value of the objective function varies greatly before and after optimization, especially in the target area, and the target value decreases by approximately $10 \%$. Meanwhile, the total number of monitor units remains the same.

As shown in Fig. 8, due to the smoothing and convexgroove effects of the fluence maps, the optimized fluence maps have a smoother distribution. Furthermore, the latter has a lower diagonal distribution than the former.

After the angle selection is completed, we smooth the fluence map by a PDE with prior knowledge and a minimization of the total number of monitor units.

After determining the optimal gantry angles, it can be determined from the comparison of the fluence maps (Fig. 9) that by considering the smooth orientation and prior information, it is easier to modulate the fluence map due to isotropic smoothing, but the smoothed map ignores the protection of the OAR. In contrast, the fluence maps obtained by our smoothing and subfield decomposition process no longer exhibit radiation from large areas, but show relatively regular radiation blocks, which better reflects the protection of the OAR and other nontarget tissues.

By comparing the dose volume histogram (DVH) and dose distribution of the target area, we can see that the dose distribution of the target area does not change significantly after the new algorithm is adopted (Fig. 10). However, by comparing the DVH and dose distribution in the nontarget area, we can see that the dose distribution in the nontarget area is obviously optimized using the new algorithm (Fig. 11). For the OAR regions such as the brain stem and spinal cord, and for the nontarget organization regions such as the mandible and other tissues, the DVH curves are very low. It can also be determined from the dose distribution map that the dose distribution obtained by the new algorithm decreases more steeply from the target area to the brainstem (Fig. 12).

It is also shown from the objective function value that the target value is essentially unchanged for the target area, while for the nontarget area, the value decreases by 16\% (Table 3). Meanwhile, the TNMU remained essentially unchanged.

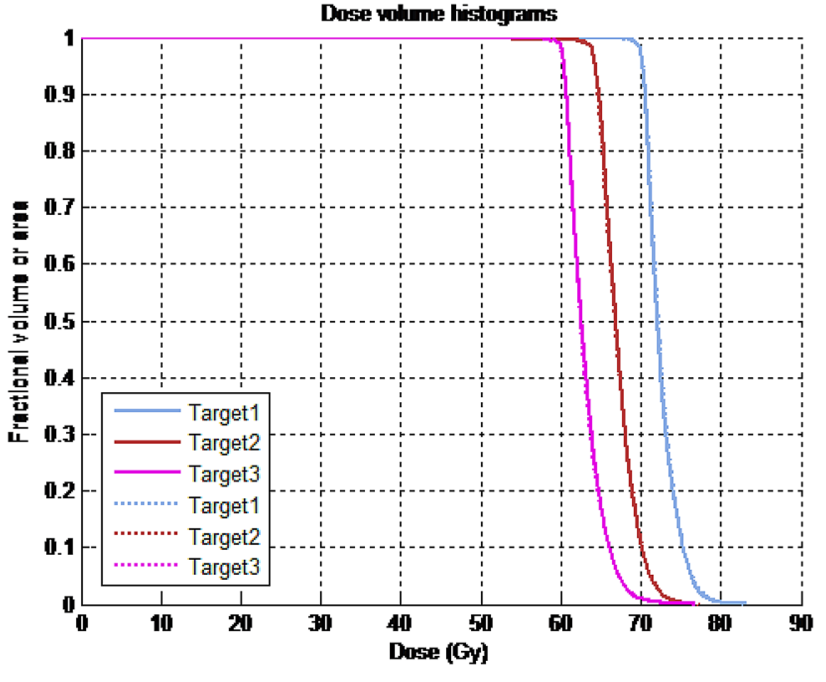

Fig. 10 Target dose volume curve comparison between conventional smoothing (solid line) and PDE-based smoothing (dotted line)

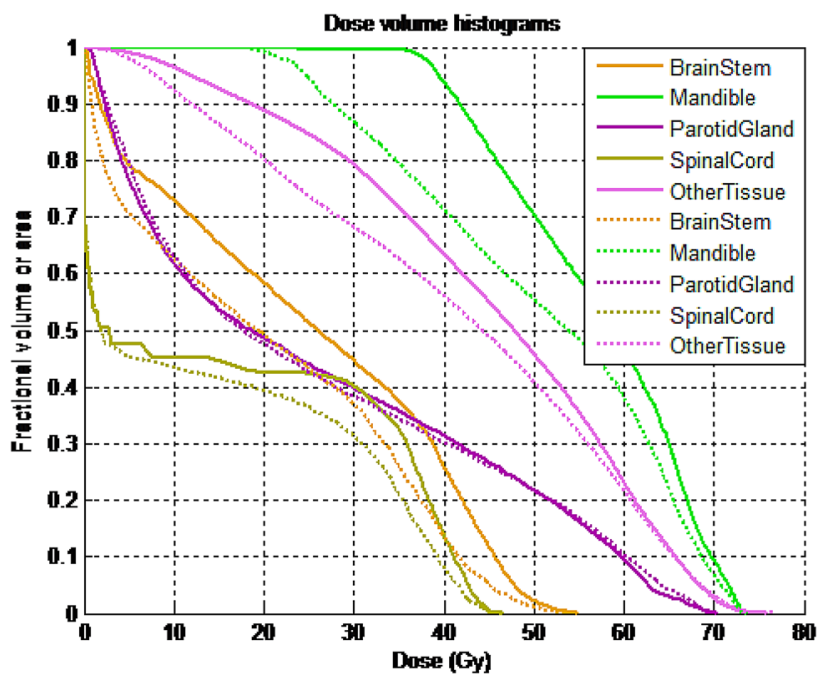

Fig. 11 Nontarget dose volume curve comparison between conventional smoothing (solid line) and PDE-based smoothing (dotted line)

\section{Conclusion}

Because the key factors of postmodulation are taken into account as much as possible in the early optimization, the intelligent IoT-based large-scale inverse planning strategy proposed in this paper has been effectively optimized in many problems, such as dose distribution optimization, gantry angle selection and fluence map smoothing. Compared with the conventional divide-and-conquer and step-by-step optimization strategies, the proposed large-scale healthcare planning method considering key factors of the postmodulation process provides an integrated solution that can also be applied to other inverse planning systems. Next, we will 


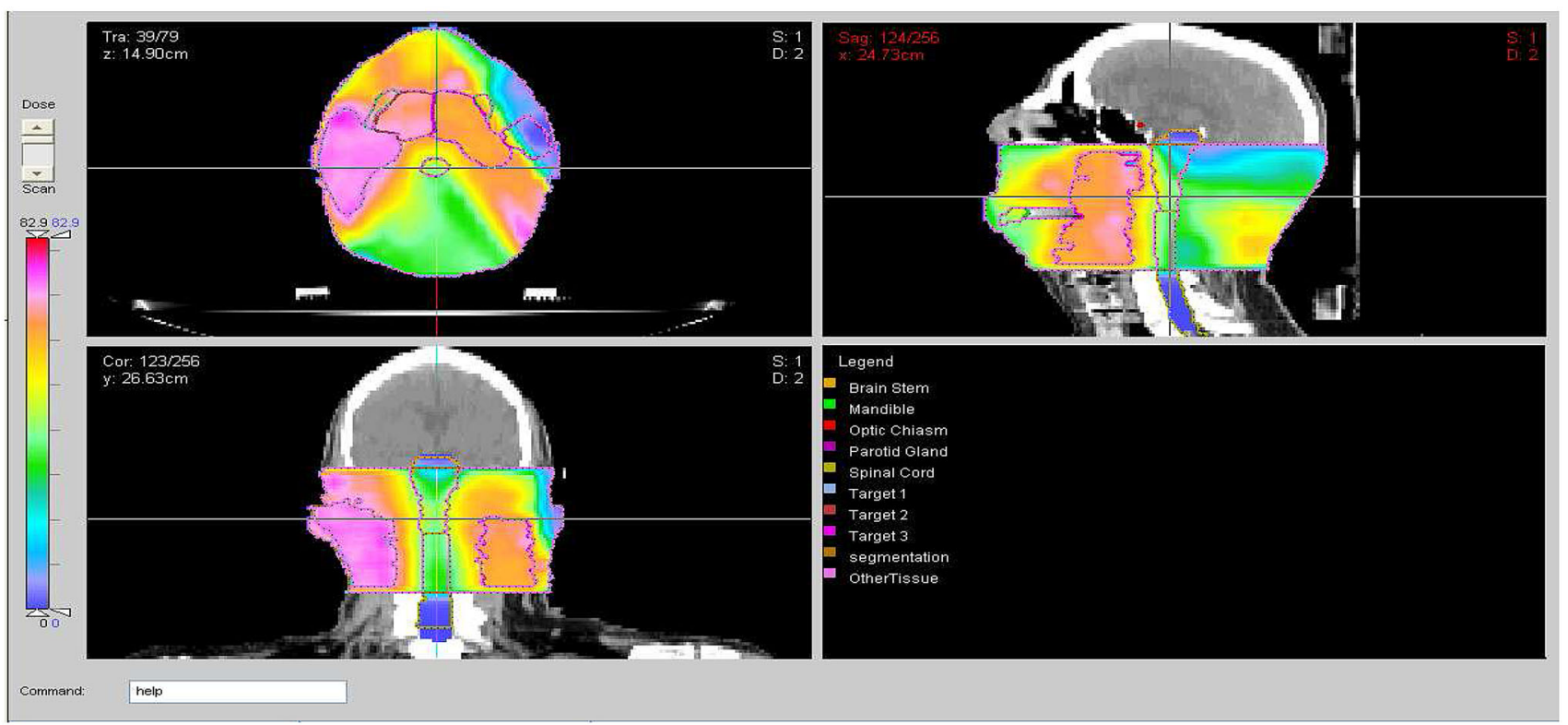

(a)

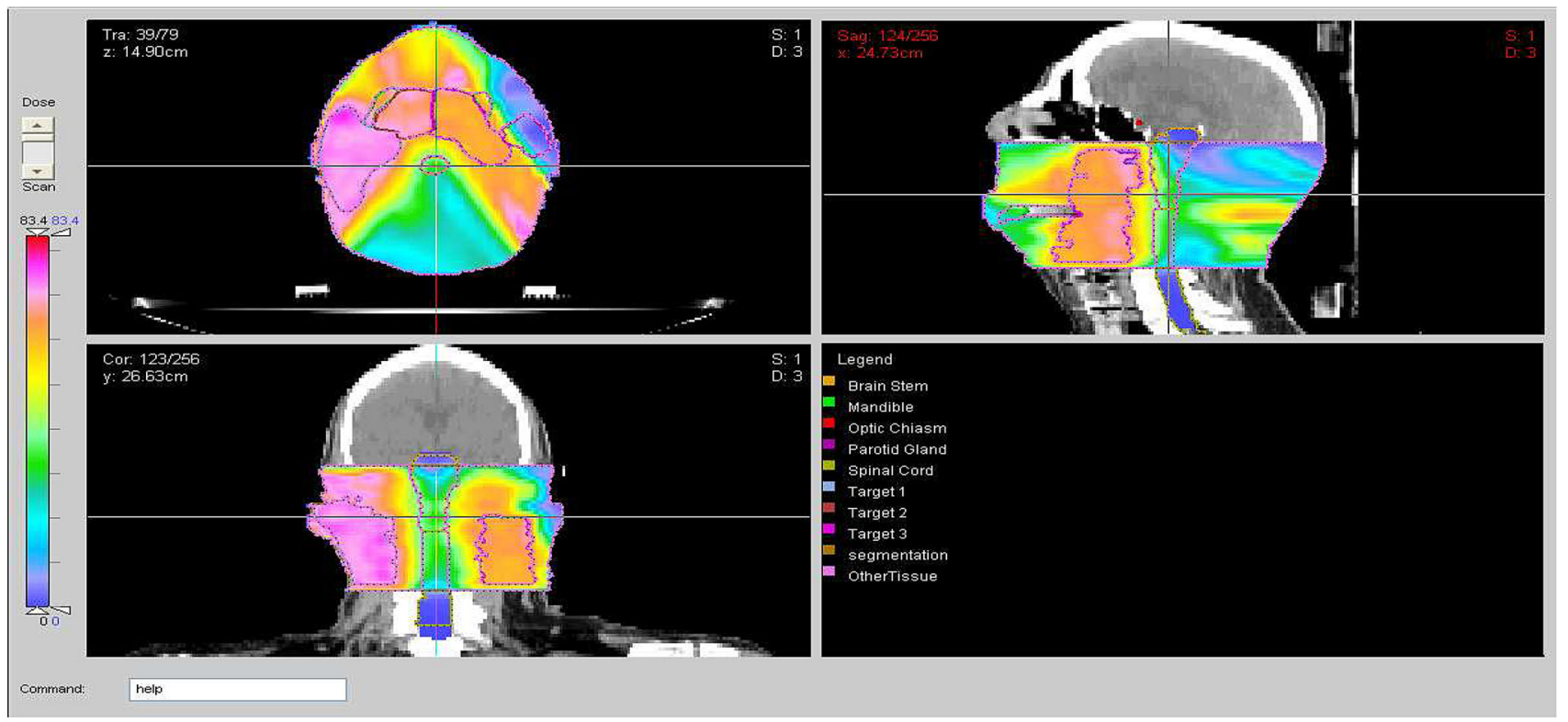

(b)

Fig. 12 Dose distribution comparison between conventional smoothing (a) and PDE-based smoothing (b)

Table 3 The objective value and monitor unit comparison after PDE smoothing

\begin{tabular}{lll}
\hline & Result after angle selection & Final result \\
\hline Objective value & 100.7484 & 88.7349 \\
Target value & 27.9765 & 28.1175 \\
Nontarget value & 72.7720 & 60.6174 \\
Monitor units & {$[22,19,35,25,11]$} & {$[25,14,36,24,11]$} \\
\hline
\end{tabular}


further consider the rapid application of medical consortium in inverse planning.

Funding Funding was provided by National Natural Science Foundation of China (61401242).

\section{Compliance with ethical standards}

Conflict of interest On behalf of all authors, the corresponding author states that there is no conflict of interest.

Open Access This article is licensed under a Creative Commons Attribution 4.0 International License, which permits use, sharing, adaptation, distribution and reproduction in any medium or format, as long as you give appropriate credit to the original author(s) and the source, provide a link to the Creative Commons licence, and indicate if changes were made. The images or other third party material in this article are included in the article's Creative Commons licence, unless indicated otherwise in a credit line to the material. If material is not included in the article's Creative Commons licence and your intended use is not permitted by statutory regulation or exceeds the permitted use, you will need to obtain permission directly from the copyright holder. To view a copy of this licence, visit http://creativecomm ons.org/licenses/by/4.0/.

\section{References}

1. Wang S-H, Zhang Y-D, Yang M, Liu B, Ramirez J, Gorriz JM (2019) Unilateral sensorineural hearing loss identification based on double-density dual-tree complex wavelet transform and multinomial logistic regression. Integr Comput Aided Eng 26(4):411-426

2. DeRoover R, Crijns W, Poels K, Nulens A, Vanstraelen B, Haustermans K, Depuydt T (2018) PO-1008: Commissioning of IMRT/VMAT on the novel Varian Halcyon. Radiother Oncol. 127:S563. https://doi.org/10.1016/S0167-8140(18)31318-5

3. Kim H, Huq MS, Lalonde R, Houser CJ, Beriwal S, Heron DE (2019) Early clinical experience with varian halcyon v2 linear accelerator: dual-isocenter IMRT planning and delivery with portal dosimetry for gynecological cancer treatments. J Appl Clin Med Phys 20(11):111-120

4. Ray X, Bojechko C, Moore KL (2019) Evaluating the sensitivity of halcyons automatic transit image acquisition for treatment error detection: a phantom study using static IMRT. J Appl Clin Med Phys 20(11):131-143

5. Wang S, Tang C, Sun J, Zhang Y (2019) Cerebral micro-bleeding detection based on densely connected neural network. Front Neurosci $13: 422$

6. Jarema T, Aland T (2019) Using the iterative kv CBCT reconstruction on the varian halcyon linear accelerator for radiation therapy planning for pelvis patients. Phys Med 68:112-116

7. Nguyen D, Pietri FJ, Sporea C, Khodri M (2018) 17 patient quality assurance of the new halcyon linear accelerator (varian). Phys Med $56: 10-11$

8. Wang S-H, Muhammad K, Hong J, Sangaiah AK, Zhang Y-D (2020) Alcoholism identification via convolutional neural network based on parametric relu, dropout, and batch normalization. Neural Comput Appl 32(3):665-680

9. Mukhtar R, Butt S, Rafaye MA, Iqbal K, Mazhar S, Sadaf T (2020) An institutional review: dosimetry comparison between simultaneous integrated boost IMRT and VMAT for prostate cancer. J Radiother Pract. 1-11. https://doi.org/10.1017/S1460396920000370
10. Qian J, Yang Y, Xing P, Wang C, Tian Y, Lu X (2020) Differences in lower cranial nerve complications predicted by the NTCP model between rtog and reduced-volume IMRT planning in radiotherapy for nasopharyngeal carcinoma. Transl Cancer Res 9(1):300-308

11. Xu D, Li G, Li H, Jia F (2017) Comparison of IMRT versus 3D-CRT in the treatment of esophagus cancer: A systematic review and metaanalysis. Medicine 96(31):e7685. https://doi.org/ 10.1097/md.0000000000007685

12. Ahmed SK, Kruse JJ, Bradley TB, Beltran CJ, Laack NNI (2018) Clinical efficacy and safety of a highly conformal, supine, hybrid forward and inverse planned intensity modulated radiation therapy technique for craniospinal irradiation. Acta Oncol 57(5):629-636

13. Wang H, Dong P, Liu H, Xing L (2017) Development of an autonomous treatment planning strategy for radiation therapy with effective use of population-based prior data. Med Phys 44(2):389396

14. Babier A, Boutilier JJ, McNiven AL, Chan TC (2018) Knowledgebased automated planning for oropharyngeal cancer. Med Phys 45(7):2875-2883

15. Rastogi M, Sapru S, Gupta P, Gandhi AK, Mishra SP, Srivastava AK, Khurana R, Hadi R, Sahni K, Farzana S (2017) Prospective evaluation of intensity modulated radiation therapy with simultaneous integrated boost (IMRT-SIB) in head and neck squamous cell carcinoma in patients not suitable for chemo-radiotherapy. Oral Oncol 67:10-16

16. Gonzalez MA, Maldonado X, Fa X, Planas J, Celma A, Pons A, Ruiz M, Mateos J, Pérez B, Alvarenga F et al (2018) Ep-2055: comparison of two correction protocols for IGRT in prostate cancer patients treated with IMRT. Radiother Oncol 127:S1125-S1126

17. Caudell JJ, Ward MC, Riaz N, Zakem SJ, Awan MJ, Dunlap NE, Isrow D, Hassanzadeh C, Vargo JA, Heron DE et al (2018) Volume, dose, and fractionation considerations for IMRT-based reirradiation in head and neck cancer: a multi-institution analysis. Int $\mathbf{J}$ Radiat Oncol Biol Phys 100(3):606-617

18. Tu P-C, Lin C-H, Lee H-Y, Shaw S, Wu C-J (2015) Dosimetric comparison of whole breast irradiation with hybrid IMRT and inverse-fields IMRT. Med Dosim 40(3):262-7

19. Penfold S, Zalas R, Casiraghi M, Brooke M, Censor Y, Schulte R (2017) Sparsity constrained split feasibility for dose-volume constraints in inverse planning of intensity-modulated photon or proton therapy. Phys Med Biol 62(9):3599

20. Hagan A, Sawant A, Folkerts M, Modiri A (2018) Multi-gpu configuration of $4 \mathrm{~d}$ intensity modulated radiation therapy inverse planning using global optimization. Phys Med Biol 63(2):025028

21. Stambaugh C, Ezzell G (2018) A clinically relevant IMRT QA workflow: design and validation. Med Phys 45(4):1391-1399

22. Interian Y, Rideout V, Kearney VP, Gennatas E, Morin O, Cheung J, Solberg T, Valdes G (2018) Deep nets vs expert designed features in medical physics: an IMRT QA case study. Med Phys 45(6):26722680

23. Liu H, Chen Y, Lu B (2018) A new inverse planning formalism with explicit DVH constraints and kurtosis-based dosimetric criteria. Phys Med Biol 63(18):185015

24. Babier A, Boutilier JJ, Sharpe MB, McNiven AL, Chan TC (2018) Inverse optimization of objective function weights for treatment planning using clinical dose-volume histograms. Phys Med Biol 63(10): 105004

25. Hashimoto S, Fujita Y, Katayose T, Mizuno H, Saitoh H, Karasawa K (2018) Field-size correction factors of a radiophotoluminescent glass dosimeter for small-field and intensity-modulated radiation therapy beams. Med Phys 45(1):382-390

26. Oconnor D, Yu V, Nguyen D, Ruan D, Sheng K (2018) Fractionvariant beam orientation optimization for non-coplanar IMRT. Phys Med Biol 63(4):045015

27. Andreozzi JM, Mooney KE, BrAuza P, Curcuru A, Gladstone DJ, Pogue BW, Green O (2018) Remote cherenkov imaging-based 
quality assurance of a magnetic resonance image-guided radiotherapy system. Med Phys 45(6):2647-2659. https://doi.org/10.1002/ mp.12919

28. Liu X, Pelizzari C, Belcher AH, Grelewicz Z, Wiersma RD (2017) Use of proximal operator graph solver for radiation therapy inverse treatment planning. Med Phys 44(4):1246-1256

29. Yuan L, Zhu W, Ge Y, Jiang Y, Sheng Y, Yin F-F, Wu QJ (2018) Lung IMRT planning with automatic determination of beam angle configurations. Phys Med Biol 63(13):135024

30. Guthier CV, Damato AL, Viswanathan AN, Hesser JW, Cormack RA (2017) A fast multitarget inverse treatment planning strategy optimizing dosimetric measures for high-dose-rate (HDR) brachytherapy. Med Phys 44(9):4452-4462
31. Dorigo M, Blum C (2005) Ant colony optimization theory: a survey. Theor Comput Sci 344(2-3):243-278

Publisher's Note Springer Nature remains neutral with regard to jurisdictional claims in published maps and institutional affiliations. 